\title{
Obscure upper GIT haemorrhage: Primary renal artery aneurysm with duodenal and inferior venocaval fistulae
}

\author{
B Puttergill, ${ }^{1}$ I Bombil, ${ }^{1}$ S Pather ${ }^{2}$ \\ ${ }^{1}$ Department of General Surgery, Faculty of Health Sciences, University of the Witwatersrand Gauteng South Africa \\ ${ }^{2}$ Department of Anatomical Pathology, Faculty of Health Sciences, University of the Witwatersrand Gauteng South Africa
}

Corresponding author: Dr B Puttergill (brookeputtergill@hotmail.com)

\begin{abstract}
Summary: We present a case of primary renal artery aneurysm presenting as obscure overt upper gastrointestinal bleeding due to an arterioenteric and arteriovenous fistula in a HIV patient not on HAART. Despite the low incidence of visceral artery aneurysms, complications and clinical presentations demand rapid intervention to avoid high reported mortality rates. We highlight the diagnostic and treatment dilemmas of this fatal complication in an immunocompromised patient.
\end{abstract}

S Afr J Surg 2018;56(3)

http://dx.doi.org/10.17159/2078-5151/2018/v56n3a2379

\section{Case report}

A 32-year-old female presented haemodynamically unstable with an upper gastrointestinal (UGIT) haemorrhage evidenced by haematemesis and fresh rectal bleeding. She had a compensated acidosis and haemoglobin of $6.9 \mathrm{~g} / \mathrm{dl}$ with a tender right upper quadrant. She was HIV positive not on highly active antiretroviral therapy (HAART) with a CD4 count of 178 and a Viral Load of $>32000000$ copies $/ \mathrm{ml}$. Her renal function indices were a urea $17.9 \mu \mathrm{mol} / \mathrm{L}$ creatinine $194 \mu \mathrm{mol} / \mathrm{L}$. She was noted to have pyuria caused by Escherichia coli. On admission she was transfused and commenced on a proton pump inhibitor and octreotide as variceal bleeding had not been excluded.

The patient responded well to resuscitation and the initial emergency endoscopy revealed a shallow oesophageal ulcer which was biopsied. There was no blood or coffee ground or site of bleeding identified. Rebleeding occurred and repeat endoscopy was negative. However, sonography for the pyuria confirmed an aneurysm in the right kidney and a collection in Morrison's pouch. Thereafter a CT angiography revealed a right renal artery aneurysm (Figure 1) with involvement of the primary and secondary branches, with air in the aneurysm sac (Figure 1) without contrast extravasation into the GIT. Prior to theatre the patient had a massive hematemesis and required emergency intubation and inotropic support. At surgery, in view of her profound hypotension, supra renal aortic clamping was performed prior to dissection to gain proximal control of the right renal artery and expose the fistula between the kidney and the duodenum which was divided and closed in two layers with polydiaxone (Figure 2). As the kidney was dissected the inferior pole was noted to communicate with the IVC. The fistula was divided and the IVC repaired with polypropylene. A septic collection was found in the retroperitoneum on the medial aspect of the renal bed which was irrigated and drained. The patient was packed due to a coagulopathy and taken with an open abdomen to ICU. She underwent 2 relook laparotomies over the next 48 hours due to persistent bloody drainage. At the latter exploration she was not actively bleeding and the packs were removed. Despite initial improvement and extubation she deteriorated due to systemic and pulmonary sepsis and died from MOF 10 days postoperatively.

She was found to be cytomegalovirus positive on serology and the oesophageal tissue demonstrated acid fast bacilli. Histology of the kidney confirmed the renal artery aneurysm and vasculopathic changes that included intimal fibrosis, focal calcification of the internal elastic lamina, inflammation in the vaso vasora and medial fibrosis. In addition, there were features of pyonephrosis and granulomatous inflammation.

\section{Discussion:}

Visceral artery aneurysms comprise a group of vascular pathologies pertinent to any emergency surgeon's clinical repertoire as they necessitate urgent intervention due to their high mortality when they erode into adjacent structures. ${ }^{1-3}$ They are primary or secondary to prior surgery ${ }^{1}$ and are morphologically divided into true aneurysms and pseudoaneurysms, features which are linked to etiology and anatomical site. The clinical presentation incorporates a wide spectrum from asymptomatic aneurysms to frank rupture (22.5-90\% dependent on site of origin). ${ }^{1-4} \mathrm{~A}$ rare primary 
visceral aneurysm is that of the renal artery, with an incidence of $0.09-0.1 \%$, which is increasingly being diagnosed incidentally due to increased use of angiographic imaging. ${ }^{2-3}$

In the larger reported series, predominantly small and asymptomatic aneurysms are included associated with hypertension, autoimmune inflammatory conditions and following reconstruction. ${ }^{2-5}$ To the authors knowledge, no literature of any case demonstrating primary renal artery aneurysm with duodenal and venocaval fistula exists, however secondary aneurysms with grafts in situ and intestinal fistula are found. The entity of HIV associated aneurysms is clinically relevant to vascular and general surgery in high HIV prevalent environments. ${ }^{6}$ This South African case report serves to recognise the dramatic presentation of a rare primary renal artery aneurysm with obscure gastrointestinal haemorrhage in an HIV positive patient.

Obscure upper gastrointestinal bleeding accounts for 5\% of all upper gastrointestinal bleeds with an overall mortality of $10 \%$. Arterioenteric fistula, despite an incidence of $0.02-0.07 \%$, have a much higher mortality rate ranging

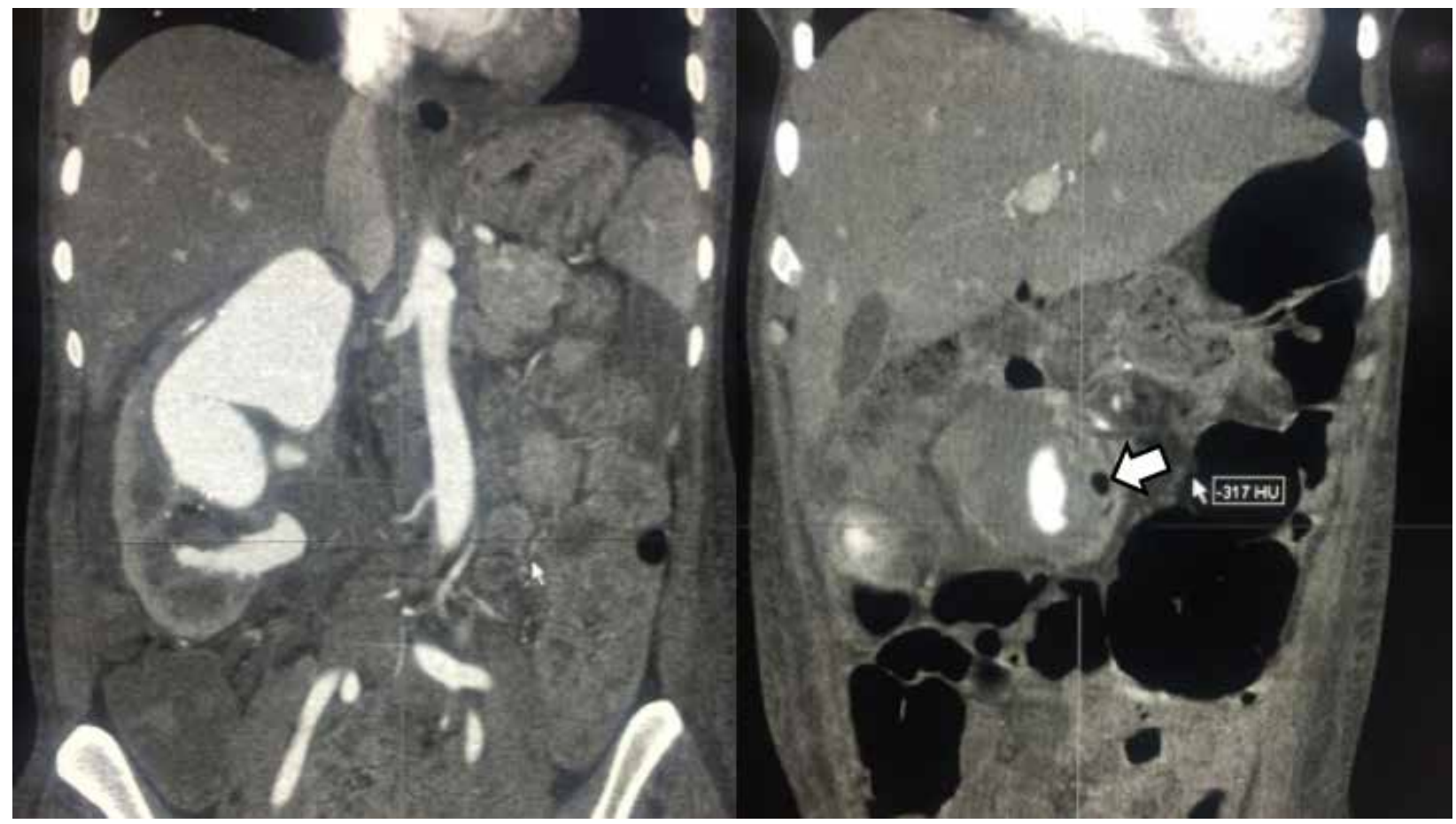

Figure 1. Abdominal Ct angiogram with renal artery aneurysm extending intrarenal with air in aneurysm (arrow) sac

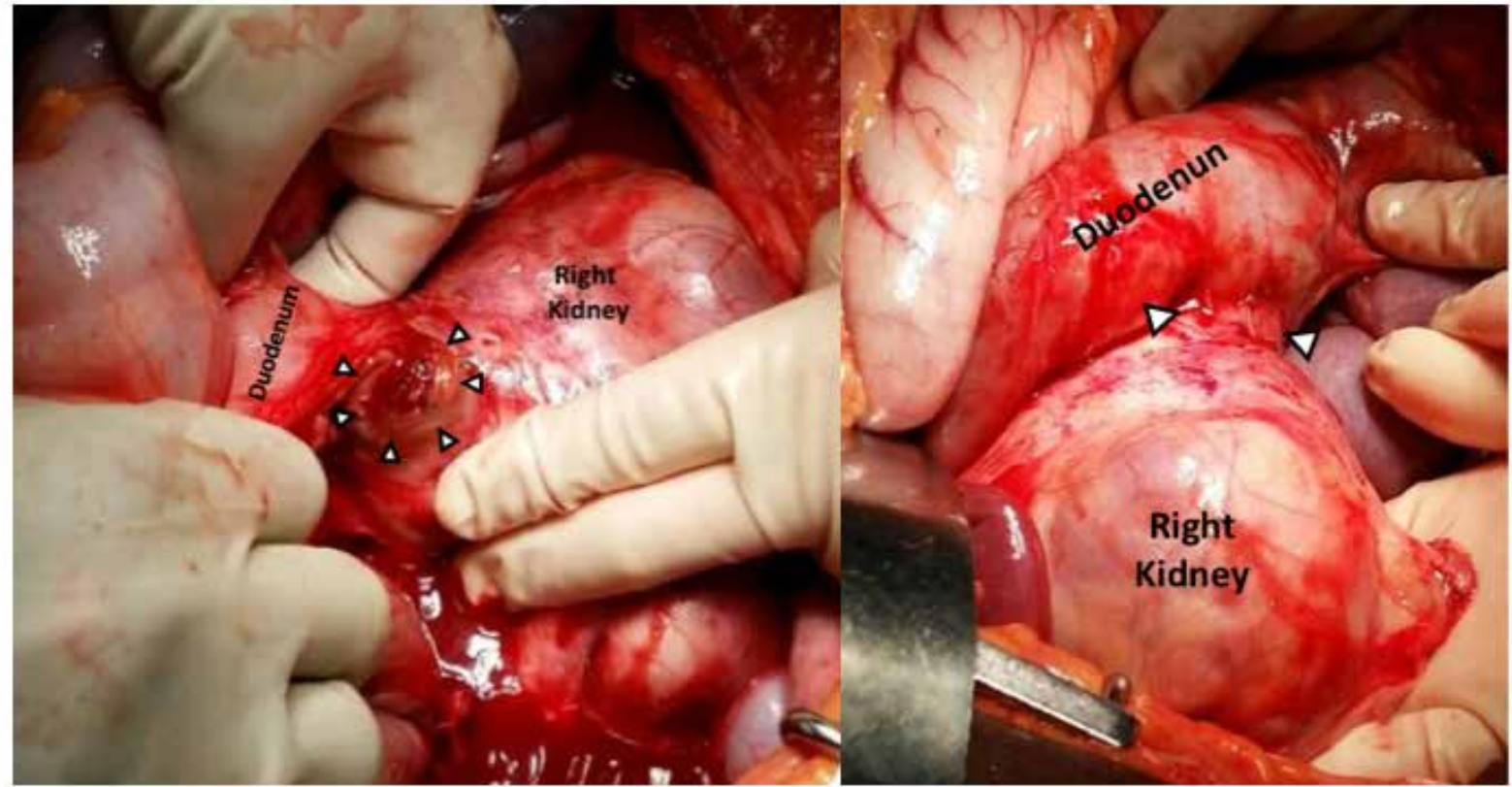

Figure 2. Opened arterioenteric tract (arrows) on anterior surface kidney and D2/D3 juntion with fistula (arrows) to ant surface of kidney 
from 20-90\%. Rapid diagnosis and intervention by a vigilant clinician is required to reduce mortality ${ }^{4,5}$ Mortality rates of elective repair of RAA remain low, however with rupture reported rates carry a $10 \%$ mortality even without an arterioenteric fistula. ${ }^{2,3}$ A systematic review revealed only 14 cases of arterioenteric fistula from the renal artery following previous grafting and surgery, however with a near $43 \%$ reported mortality, and highlights that the clinician must have a high degree of suspicion to pursue diagnosis and therapy rapidly if the patient is to survive.

HIV-associated vasculopathy tends to manifest in young patients and has a predilection for medium and large vessels. ${ }^{6}$ HIV seropositive patients may present with aneurysmal disease at atypical sites as a result of perivasculitis in the vasa $^{5}$ vasorum with inflammatory infiltrate into the vessel wall. Nair et al. further contributed that necrosis, luminal narrowing, fibrosis, loss of medial elastic fibres or smooth muscle and fibromuscular hyperplasia may also be evident. Consequently a variety of clinical presentations may occur. ${ }^{6}$

This patient highlights the problems of practice in a high HIV prevalent environment - profound immunosuppression, opportunistic infection, complex arteriovenous fistulae surgery and hypotension - which resulted in a turbulent postoperative course and mortality.

\section{REFERENCES}

1. Chadha M, Ahuja C. Visceral artery aneurysms: Diagnosis and percutaneous management. Semin Intervent Radiol. 2009;26(3):196-206. doi: 10.1055/s-0029-1225670. ISSN 0739-9529

2. Henke PK, Cardneau JD, Welling TH, Upchurch GR, Wakefield TW, Jacobs LA, et al. Renal artery aneurysms: a 35-year clinical experience with 252 aneurysms in 168 patients. Ann Surg. 2001;234(4):454-62-3. PMCID: PMC1422069

3. Wason SEL, Schwaab T. Spontaneous Rupture of a Renal Artery Aneurysm Presenting as Gross Hematuria. Review Urology. 2010;12(4):e193-e196. doi: 10.3909/riu0489

4. Bergqvist D, Björck M. Secondary Arterioenteric Fistulation A Systematic Literature Analysis. Eur J Vasc Endovasc Surg. 2009;37(1):31-42. doi:10.1016/j.ejvs.2008.09.023

5. Bergqvist D, Björck M. Enteric fistulation after renal artery reconstruction: A systematic literature review. World J Surg. 2010;34(10):2305-7. doi: 10.1016/j.ejvs.2016.09.014

6. Nair R, Abdool-Carrim A, Chetty R, Robbs J. Arterial aneurysms in patients infected with human immunodeficiency virus: a distinct clinicopathology entity? J Vasc Surg. 1999;29(4):600-7. doi: 10.1016/S0741-5214(99)70304-6 